\title{
Enhanced tensor factorization framework using non-negative and probabilistic tensor factorization approaches for microblogging content propagation modelling
}

\author{
N. Baggyalakshmi ${ }^{*}$, A. Kavitha ${ }^{2}$, A. Marimuthu ${ }^{3}$ \\ ${ }^{1}$ M.Phil, Department of Computer Science, Kongunadu Arts and Science College, Coimbatore. \\ ${ }^{2}$ Assistant Professor, Department of Computer Science, Kongunadu Arts and Science College, Coimbatore. \\ ${ }^{3}$ Assistant Professor, Department of Computer Science, Government Arts and Science College, Coimbatore. \\ *Corresponding author E-mail: baggyanethra@gmail.com
}

\begin{abstract}
With the aim of identifying the user's preferences, Content propagation modeling from the micro-blogging sites aids diverse organizations. In existing studies, four user behavior aspects were used by the content propagation model that is to say topic virality, user's position, user susceptibility and user virality. The propagation occurrences are signified as a tensor factorization model so-called V2S is presented with the aim of deriving the behavioral aspects via which the content propagation is designed. On the other hand, it doesn't comprise the linguistic patterns in the content that decreases the performance of the content propagation. Moreover, by utilizing advanced tensor approaches, the factorization structure is improved. Therefore the performance of the complete system is decreased meaningfully. With the aim of overcoming the aforesaid problems, Enhanced V2S (EV2S) Tensor Factorization framework is presented in this research that make use of the Probabilistic Latent Tensor Factorization (PLTF) as well as Non-negative Tensor Factorization (NTF) in order to derive the behavioral facets. NTF is presented for decreasing the content propagation errors. By making use of fast gradient descent technique, the unrestrained issue, which happens in this model is solved. This research system identifies the reposts as well as re-tweets in huge datasets proficiently with minimum processing time. From the experimentation outcomes, it is proved that the EV2S-PLTF tensor factorization performs better when compared to the previous tensor frameworks.
\end{abstract}

Keywords: Content propagation, behavior factors, tensor factorization, topic virality, user virality, NTF, PLTF

\section{Introduction}

It is probable to contain large volume of data for examining with the improvements in the computational power. We have to utilize proficient techniques for extracting valuable info from that data. The matrix factorization had main effect on non-negative matrix factorization, clustering, collaborative indexing, and latent semantic indexing, in addition to numerous other approaches while regarded as matrix factorization issues. The benefit of matrix factorization depends on its fundamental structure, a matrix that lets higher parallelism in regard to computation. As well, matrix computations are well- known algorithms that are consistent, effective as well as satisfactory [1].

With the notion of finding out and excerpting unseen structure in the provided data, Tensor Factorization (TF) is utilized on multiway investigation. Numerous models are arose as there are many means to factorize a matrix. E.g., non-negative matrix factorization (NMF), canonical decomposition (CP), NMFD and NMF2D are generally used tensor factorization models. TF is an outstanding candidate for the domains that is capable of guesstimating missing values when guessing the parameters of the provided model. For performing this, initially, the point guesstimate of the values must be computed. On the other hand, as the correlation amid diverse dimensions is provided by the model itself, the model utilized for TF turn out to be very significant.

The notion of social info propagation is traced back to the idea of viral marketing [2] that takes that notions are propagated by the "word of mouth" in the marketplace. In addition, the info and influence are spread very forcefully through the Internet when compared to the conventional channels. This is stated by Scott [3]. The statement is more appropriate nowadays, and we must thankful to the rise of social networking services. Numerous politicians as well as advertisers exploit Twitter, Face book and other social media services for promoting themselves. Nowadays, for extracting the messages content and get info regarding the social links amid users, a lot of micro-blogging services offer APIs, which facilitate developers.

With the purpose of identifying the correlations amid various communities, Sun et al. [4] make use of data from the Facebook; and for building the associations amid people for examining user conducts in online, micro-blogging social networks Kwak et al. [5] utilize Twitter data. Instead, numerous current researches have concentrated on creating models to imitate influence propagation activities and are assessed by means of some features of the actual propagation phenomenon ([6], [7], [8]). The diverse influence propagation models' performance is matched up deprived of a 
unified evaluation metric. So, provided some kind of diffusion data, it is very hard to choose which influence propagation model is the most appropriate one with equivalent parameters.

A novel restrained based Stochastic Gradient Descent (SGD) solution for the extensive least square issue is examined in [9]. It uses the averaging hyper-plane as the anticipated hyper-plane. Particularly, it defined the convergence rates as well as the regret bounds for this technique. Constrained SGD works similar to a full second order method on the other hand with easy computation compared to 2SGD. In Constrained SGD, the optimal regret O (log $\mathrm{T}$ ) is attained, while adopting an equivalent learning rate approach By means of experimental outcomes, the hypothetical advantages are discussed.

The Stanford analyzer is constructed on Recursive Neural Tensor Network RNTN utilized and it gets the input phrases and signify via word vectors as well as parse tree, after that by means of the similar tensor based composition function, calculate vectors for higher nodes in the tree. Not like bag of words methods, it precisely takes the emotion by providing negative outcomes for the denial of positive phrases. Principally, this research was concentrated on compositionality in vector spaces, semantic vector spaces, deep learning, logical form, and sentiment analysis. The emotion of every tweets got from this analyzer would be kept in database.

On the other hand, the particular feature doesn't comprise the linguistic patterns in the content that decreases the performance of the content propagation. This study concentrated on giving positive and typically sparse common factors or hidden (latent) components with physical meaning and interpretation. For deriving the four behavior features, the research method is Enhanced V2S framework comprises Probabilistic Latent Tensor Factorization (PLTF) and Non-negative Tensor Factorization (NTF). Therefore the propagation prediction is improved meaningfully.

\section{Related work}

Former methods for excerpting particular info from HTML Web documents date back to the late nineties and are today usually called wrapper induction systems [10]. Numerous wrapper induction systems work in a partially automated way, need physical labeling of appropriate passages for every site template, that is to say that the product pages on Amazon.com (http://www.amazon.com) Therefore, from examples, wrappers are learned inductively. Normally, GUIs encourages the labeling process [11].

In [12], Lerman et al initially noticed the dissimilarities amongst propagation behaviors of the micro-blogging users' and those of other social systems and suggested that data could spread improved in micro-blogging networks. In [13], Wu et al, identified a two-step procedure that the data originating from the media broadcast in Twitter: (1) the data transfers from media to an intermediary layer of "opinion leaders", (2) the data transfers from the users in middle layer to their group who are less related to the media.

In [14], Suh et al explained that the survival of URLs as well as hash tags in a tweet contain robustly association with its retweetability, when the number of previous tweets of a user doesn't impact the user's likelihood of retweeting. In [15], Petrovi et al recurrent this conclusion in addition it sets up that authors' reliability contains hopeful properties on a tweet's retweetability. Later, in [16], the affirmative consequences of authority features such as constancy, proficiency, and attraction was explained by Liu et al.

In [17], Romero et al found that Twitter users illustrate various conducts while spreading hash tags of various sort as well as topics. They differentiated the behavior dissimilarities amid users by the likelihood that a user presumes a hash tag subsequently repetitive experience to the hash tag. In conscientious, they identified that the approval of diplomatically provocative hash tags is chiefly extravagant by many recurrent revelations. On the contrary, such recurrent revelations contain minimum consequence on the acquiescence of conversational hash tags.
In 2012, Mukherjee et al utilized an insubstantial technique for making use of discourse associations for polarity identification of tweets. This technique is focused in the direction of the web-based applications, which handle unstructured text, noisy text, such as the tweets, and could not meet the expense of using heavy linguistic resources such as parsing because of recurrent failure of the parsers to deal with noisy data. Numerous works in micro-blogs, such as Twitter, utilize a bag-of-words model, which disregards the discourse elements such as since, but, although and so on. It indicates how the discourse associations such as the connectives as well as conditionals are utilized to integrate discourse info in any bag-of-words method, for enhancing sentiment classification accurateness. Discourse associations and equivalent rules are found with minimal processing - simply a list search. It initially provides a linguistic explanation of numerous discourse associations that brings about conditions in rules and features in SVM. It proves that our discourse-based bag-of-words model works fine in a noisy medium (Twitter) and it does superior to previous Twitter-based application.

In 2005 Shashua et al utilized techniques for identifying a positive $\mathrm{n}$-dimensional tensor factorization (n-NTF) that comprises the nonnegative matrix factorization (NMF) as a specific instance. It inspires the usage of n-NTF in 3 data analysis fields: (i) association to latent class models in statistics, (ii) sparse image coding in computer vision, and (iii) model selection issues. It derives a"direct" positive-preserving gradient descent algorithm in addition to an alternating approach dependent upon recurrent numerous rank-1 issues.

\section{Proposed methodology}

In the research method, user virality, the topic virality, behavioral factors, linguistic features, and user susceptibility factors proficiently identify the content most subsequently to the users. Therefore the features are explored as well as it is identified that the user's location in the network and as well acts an important role to identify the content type and user behavior dependent upon it.

\subsection{Linguistic features in V2S tensor factorization}

In the entire eight features, four of them are linguistic naturally, signifying they consider syntax and distribution of characters or tokens:

Average sentence length: It is apparently greater for informative content blocks compared to clutter. For example, text within link lists is fairly limited in regard to sentence length.

Number of sentences: It is known as Continuous text, for example, news articles, inclined to be made up of numerous sentences. Therefore, informative text blocks are anticipated to fulfil the corresponding threshold by revealing superior values.

Character distribution: Working on simple characters more willingly than the token level or sentence level, the feature at hand gauges the proportion of alphanumeric characters in the text block as contrasting to non-alphanumeric characters. The assumption is that content blocks contain a greater proportion for numbers and letters.

Stop-word ratio: Stop-words are the terms, which comes more often in text and accept no intrinsic information gain. Examples in English are she" or "this". It calculates the proportion of stop-words in regard to nonstop-words by utilizing stop-word lists from 14 languages. After that It takes the argmax of all 14 ratios that provides us as a by-product the language the text is written in

The improved V2S framework comprises $(\mathrm{u}, \mathrm{v}, \mathrm{m}, \mathrm{p})$ here $\mathrm{m}$ is a known as a content item produced by user $u$ at position $p$ of $u$, and open to user $\mathrm{v}$ at position $\mathrm{p}$ of $\mathrm{v}$. The binary variable $\delta_{\text {uvmp }}$ represents if the user $\mathrm{v}$ accepts the $\mathrm{m}$ content i.e., $\delta_{\text {uvmp }}=0$ or 1 based upon the positive or negative propagation observation. The improved V2S framework utilizes $\delta_{\text {uvmp }}$ dependent upon topic virality and users position $\mathrm{p}$ in the network, topic specific susceptibility of $v$ the topic-specific virality of $u$. While taking a 
propagation observation ( $\mathrm{u}, \mathrm{v}, \mathrm{m}, \mathrm{p})$, the probability is supposed that $\mathrm{v}$ accepts $\mathrm{m}$ is by (i) $\mathrm{m}$ 's topic distribution $D_{m}=$ $\left(D_{m, 1}, \ldots, D_{m, K}\right)$, (ii) u's topic specific virality $V_{u}$, (iii) topic virality I; (iv) v's user susceptibility $S_{v}$ and (v) position of user in the network.

In the improved V2S framework, by means of higher informative content, the average sentence length is computed. Character distribution, the number of sentences, and stop word ratio are taken in the linguistic features

The stochastic gradient descent (SGD) technique is a radical simplification. Rather than calculating the gradient of En(fw) accurately, every iteration guesstimates this gradient based on a solitary arbitrarily chosen example zt :

$w_{t+1}=w_{t}-\gamma_{t} \nabla_{w}\left(z_{t}, w_{t}\right)$

The stochastic process $\{w t, t=1, \ldots\}$ based upon the samples arbitrarily chosen at every iteration. As the stochastic process doesn't want to recollect which examples were visited for the period of the former iterations, it could process examples on the fly in a deployed system. In those instances, the stochastic gradient descent unswervingly enhances the anticipated risk, as the examples are arbitrarily drawn from the ground-truth distribution. For dipping the computational complexity by less number of iterations, SGD is utilized. Therefore the searching ability is enhanced more willingly than the former technique ideally. The tensor factorization process' speed of is enhanced. Learning speed doesn't based upon the statistical estimation rate while leaning speed based upon the condition number and scales well. The algorithm provided the finest computational and content propagation model.

\section{Algorithm}

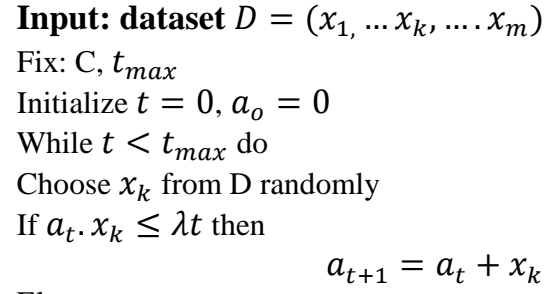

Else

$$
\begin{gathered}
a_{t+1}=a_{t} \\
t \leftarrow t+1 \\
w_{t}=a_{t} / \lambda t
\end{gathered}
$$

By cycling recurrently via the probably arbitrarily permuted training dataset and by means of the final hypothesis for prediction, this is accomplished. This process of providing the training data to the algorithm in whole eras contains in our instance, the extra benefit that there is a lower bound on the ideal value of the objective function to be reduced that is stated in regard to quantities existing all through the run. The presence of such a lower bound gives an approximation of the comparative accurateness attained by the algorithm.

Therefore, the unrestrained optimization issue is solved by means of NTF algorithm and the linguistic features are learned from the content.

For deriving the user behavior, the improved V2S tensor factorization framework uses the Probabilistic Latent Tensor Factorization (PLTF) and NTF. The NTF and PLTF are elucidated in the subsequent segments.

\subsection{Non-negative tensor factorization (NTF)}

The five behavioral factors are Factorization gauged utilizing Probabilistic Latent Tensor Factorization (PLTF) and Non-negative Tensor Factorization (NTF)
When the dataset is signified in the tensor form, to carry out a dimensionality reduction on the data, it could use tensor decomposition methods. It concentrated on the Non-negative Tensor Factorization (NTF) that is provided by the PARAFAC/CANDECOMP decomposition with non-negative restraints. NTF estimates the tensor $\mathrm{X}$ into the summation of rankone tensors, known as components:

$\mathrm{x} \approx \sum_{r=1}^{R} \lambda_{r} a_{r} b_{r} c_{r} d_{r} e_{r}$

Here, the rank of the tensor is denoted by $\mathrm{R}$, the values of the tensor core is denoted by $\lambda_{\mathrm{r},} \mathrm{L}=\operatorname{diag}(\lambda)$, and the outer product $\mathrm{a}_{\mathrm{r}}, \mathrm{b}_{\mathrm{r}}, \mathrm{c}_{\mathrm{r}}$, $d_{r}$ and $e_{r}$ denotes the component $r$, with $r=1, \ldots, R$. The vectors $\mathrm{ar}_{\mathrm{r}}, \mathrm{b}_{\mathrm{r}}, \mathrm{c}_{\mathrm{r}}, \mathrm{d}_{\mathrm{r}}$ and $\mathrm{e}_{\mathrm{r}} \quad$ correspondingly give the level of membership of the users to the component $r$, the level of membership of the features to the component $r$, and the temporal activation of the component $r$.

These vectors are encrypted in five behavioral matrices known as factor matrices. So, the guesstimate in Eq.(1) is revised in the Kruskal form

$$
\mathrm{X} \approx|\mathrm{L}, \mathrm{A}, \mathrm{B}, \mathrm{C}, \mathrm{D}, \mathrm{E}|
$$

For getting the factor matrices, and therefore the estimated tensor, must resolve an optimization issue of the form:

$$
\begin{gathered}
\min \|\mathrm{X}-[\mathrm{L}, \mathrm{A}, \mathrm{B}, \mathrm{C}, \mathrm{D}, \mathrm{E}]\|_{F}^{2} \\
\text { s.t. } \lambda, \mathrm{A}, \mathrm{B}, \mathrm{C}, \mathrm{D}, \mathrm{E} \geq 0
\end{gathered}
$$

Here $\|.\|_{F}$ is known as the Frobenius norm, and non-negativity restraints are enforced on the factor matrices.

For examining the appropriate amount of components, that is to say rank, the research method utilizes the Core Consistency Diagnostic (CORCONDIA). This technique lets for calculating the core consistency value for every simulation.

Specially, the Core Consistency (CC) values give an assessment of the nearness of the calculated decomposition to the ultimate one. By comparing the core tensor $\mathrm{L}$ of the decomposition to a superdiagonal tensor $\mathrm{G}$, this is accomplished, that is to say that a tensor containing entries equivalent to 1 on the diagonal and 0 or else

$$
\mathrm{CC}=100\left(1-\frac{\sum_{l=1}^{R} \sum_{m=1}^{R} \sum_{n=1}^{R}\left(\lambda_{l m n}-g_{l m n}\right) 2}{R}\right.
$$

Here, $\lambda_{l m n}$ are known as the entries of the core tensor $L \in R$ and $g_{l m n}$ are the entries of the ideal core $\mathrm{G} \in \mathrm{R}{ }_{\mathrm{R} \times \mathrm{R} \times \mathrm{R} . .}$ The core consistency contains an upper bound: its values could not surpass 100. Consequently, greater values of the core consistency signify greater resemblance amid the cores, when less values specify that the model chosen is difficult. Lastly, the core consistency could presume negative values, therefore representing that the model chosen is unsuitable.

When the amount of components and the equivalent factor matrices are found, the system could examine the information given by the factor matrices. Lastly, the system could get better the temporal activation of every component in the columns of the factor matrix. This info is utilized to examine the progression of every excerpted behavior [20].

\subsection{Probabilistic latent tensor factorization (PLTF)}

PLTF [21] is a framework for computing the KL and EU cost functions by means of full Bayesian inference for any tensor factorization duplication. The authors conquered the dualism amongst exponential families as well as Bregman divergences that allowable them to hypothesize the TF crisis into an inference crisis of a probabilistic graphical duplication with Gaussian or Poisson technique that reduce to a factor estimation issue. Authors as well discard to message passing to added reduce the replication into a series of primitive matrix process. With the aim of achieving this, they started a notation that is identical to undirected probabilistic 
graphical duplications for TF. This notation produces the replication useful for numerous application domains identical to network investigation, audio processing, collaborative filtering or vision. All of them must be anticipated mainly for the application domain.

PLTF generative model is discrete as:

$$
\begin{aligned}
& \Lambda(w)=\prod_{\alpha}^{N} Z_{\alpha}\left(w_{\alpha}\right) \text { intensity } \\
& S(w) \sim P O(S ; X(w)) K L \text { cost } \\
& X\left(w_{0}\right)=\sum_{w_{0}}^{S(w) \text { observation }} \\
& \hat{X}\left(w_{0}\right)=\sum_{\bar{w}_{0}} \Lambda(w) \text { parameter } \\
& M\left(w_{0}\right)= \begin{cases}0 & X\left(w_{0}\right) \text { is missing } \\
1 & \text { otherwise }\end{cases}
\end{aligned}
$$

In the replication, $\Lambda(w)$ is denoted as latent intensity field. Because of the reproductive possessions of the Poisson distribution, the observation $X\left(w_{0}\right)$ as well as the cost $S(w)$ contain identical type of distribution. Consequently the crisis of minimization the distance amongst investigation and valuation turn out to be maximization oflog $\left(p\left(X \mid Z_{1: n}\right)\right)$. The missing standards in the observation replica is hold by the probability maximization:

$$
p(X, S \mid Z)=\prod_{w}\left(p\left(X\left(w_{0}\right) \mid S(w)\right) p(S(w) \mid \Lambda(w))\right)^{M\left(w_{0}\right)}
$$

Grounded on the user behavior parameters, $l\left(\delta_{u v m p}\right)$ is resultant through PLTF as:

$$
\begin{aligned}
l\left(\delta_{u v m p}\right)=\mu(u, v, m, p)^{\delta_{u v m p}} .(1 \\
-\mu(u, v, m, p))^{\left(1-\delta_{u v m p}\right)}
\end{aligned}
$$

The loss function $R_{l, f}(u, v, m, p)$ is computed as

$$
\begin{aligned}
R_{l, f}(u, v, m, p)=- & \delta_{u v m p} \cdot \ln (\mu(u, v, m, p)) \\
& -\left(1-\delta_{u v m p}\right) \cdot \ln (1-\mu(u, v, m, p))
\end{aligned}
$$

Subsequent to admission the $l\left(\delta_{u v m p}\right)$ and the loss function $R_{l, f}(u, v, m, p)$, the valuation of $L(I, V, S, P)$ is implemented through the fast gradient as mentioned over to build a procedure pool and the computation procedure for $\sum_{(u, v, m, p) \epsilon \mathrm{O} u} R_{l, f}(u, v, m, p)$. The process pool is subjugated to reduce the running time in computing the gradients and rereading the variables mainly for greater tweet datasets.

\section{Performance evaluation}

The performance of the presented enhanced V2S (will be denoted as EV2S) framework is assessed in this part, and evaluations are done by comparing with the previous V2S framework. The performance parameters called Area under the Precision Recall Curve (AUPRC), precision (@10\%) and running time are utilized. The evaluations are done amid previous V2S-based probabilistic factorization model (V2S-PF), V2S-based numerical factorization model (V2S-NF) and the M-V2S-based HOSVD (MV2S-HOSVD) and M-V2S based PLTF (MV2S-PLTF) framework models.

\subsection{Dataset}

The Twitter dataset (as utilized in [22]) is a huge quantity of tweets gathered previously the 2012 US presidential election. With the aim of building this corpus, initially a set of 56 seed users are physically chosen. These are greatly-followed as well as politically-concerned Twitter users, comprising main US politicians, for example Mitt Romney, Barack Obama, and Newt Gingrich; famous political bloggers, for example Red State, America Blog and Daily Kos; and political units of US news media, for example CNN Politics, and
Huffington Post Politics. The group of users was extended by including each and every users subsequent to no less than three seed users with the aim of obtaining numerous politics savvy users. Finally, the subsequent network amongst those users and all their tweets posted for the period of the first two weeks of October 2012 are crawled. This duration comprises numerous events associated with the 2012US presidential election, for example, the national conventions of democratic as well as republican parties, and the debates amid presidential candidates. This dataset comprises network as well as content propagation for a huge set of Twitter users' vigorously contributing US politics for the time of a politically active period. So, we guess tweets in this dataset to be finely read, and greatly retweeted. The crawled data spans over 14 days, split it into 10 sliding windows: every window spans 5 days, and the sliding step is 1 day. Approximately, in every time window, roughly $4 \%$ of tweets are retweeted and every tweets produced about 3.5 retweets, bringing about $14 \%$ of the entire tweets are retweets.

\subsection{Comparison results}

\section{AUPRC}

Area under the Precision Recall Curve (AUPRC) for the previous and presented approaches are matched up with the global retweet prediction task as well as personalized retweet prediction task.

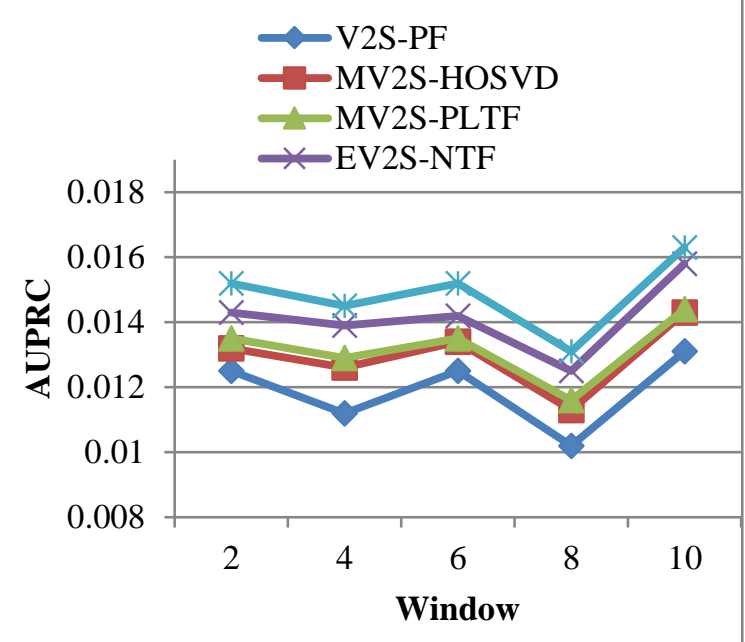

Figure 1a: Global retweet prediction task

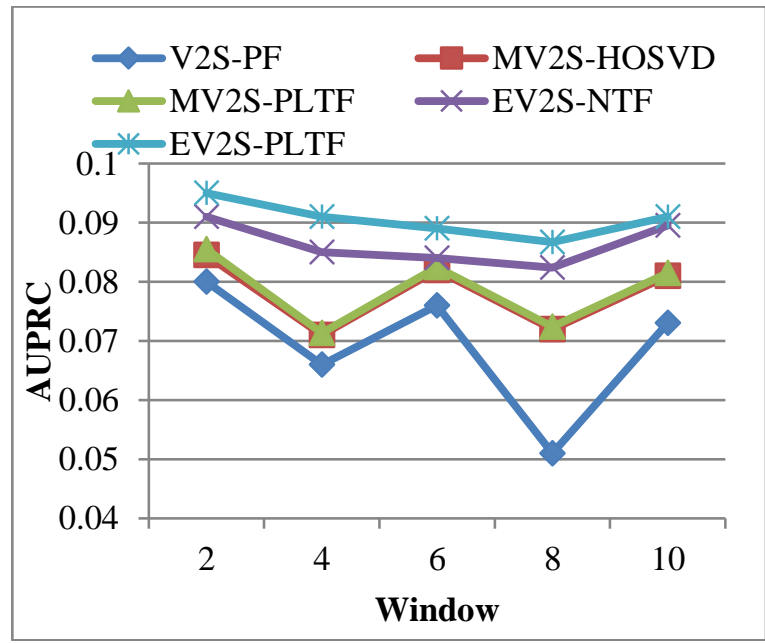

Figure 1b: Personalized retweet prediction task

Figure 1 a) \& b) depicts the evaluation of V2S, MV2S and EV2S frameworks for global and personalized retweet prediction activities correspondingly in regard to AUPRC. According to the graphs, it is proved that the presented EV2S-PLTF, EV2S-NTF based factorization approaches does superior to the MV2S and V2S based factorizations with greater values of AUPRC. 


\section{Precision}

Precision of the factorization models are associated for example topic distribution for every user that is completely skewed to $10 \%$ of overall $\mathrm{K}$ topics with the aim of creating every user's tweets concentrates on just certain topics.

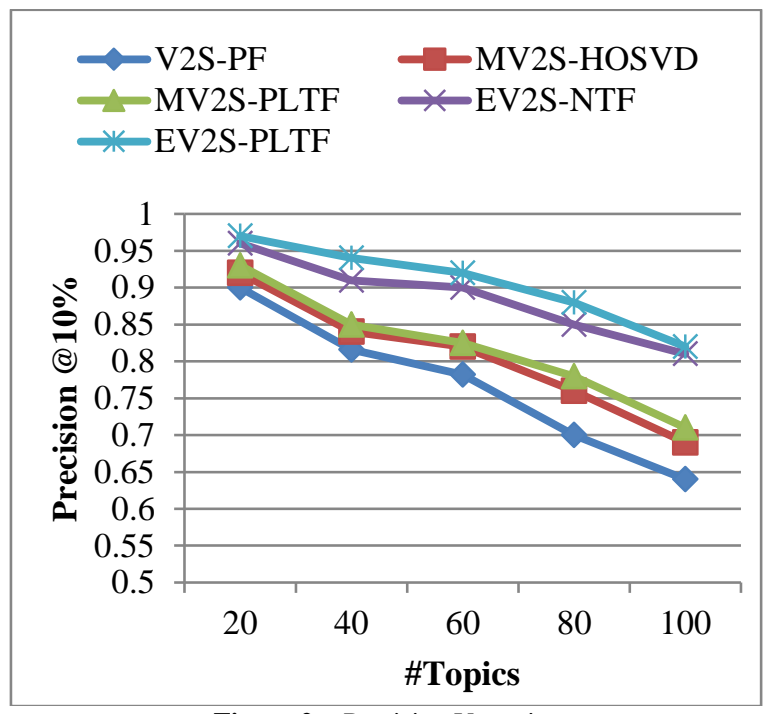

Figure 2a: Precision Vs topics

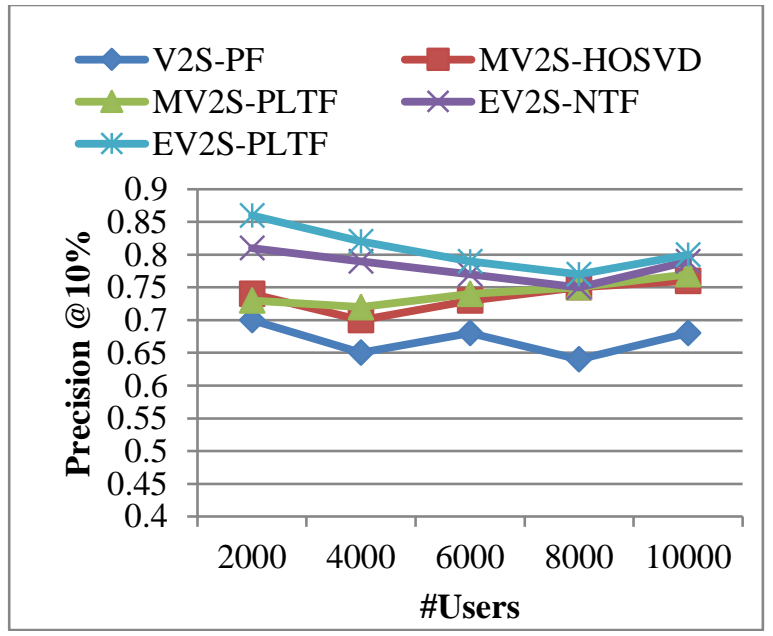

Figure 2b: Precision Vs users

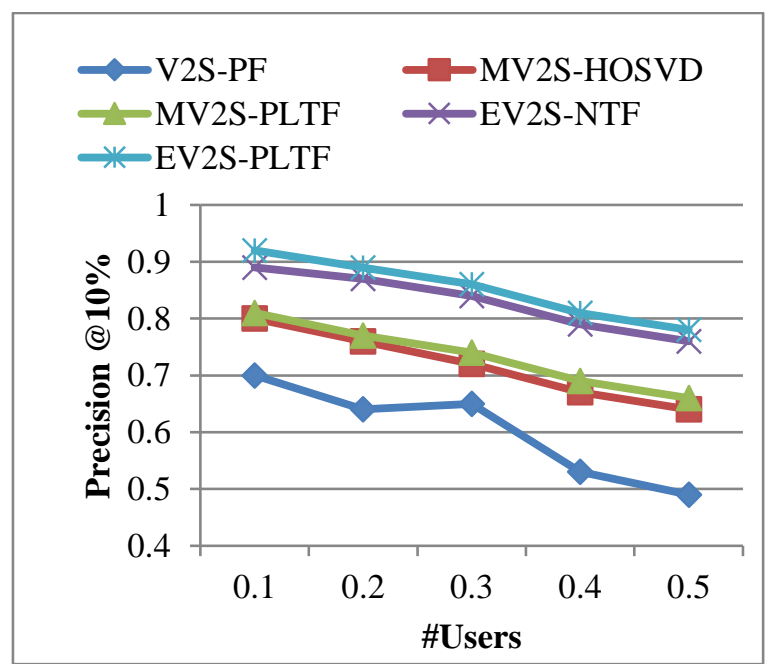

Figure 2c: Precision Vs Score width

Figure 2 a), b), c) depicts the evaluation of the factorization approaches in regard to precision (@10\% topics) regarding topics, users and score width correspondingly. According to the graphs it is identified that the presented EV2S-NTF, EV2S-PLTF based factorization gives greater precision values compared to the previous MV2S and V2S based approaches. Therefore the effectiveness of comprising linguistic features as the fifth behavior factor is justified.

\section{Runtime}

The runtime of the factorization frameworks are matched up with the amount of users and the topics.

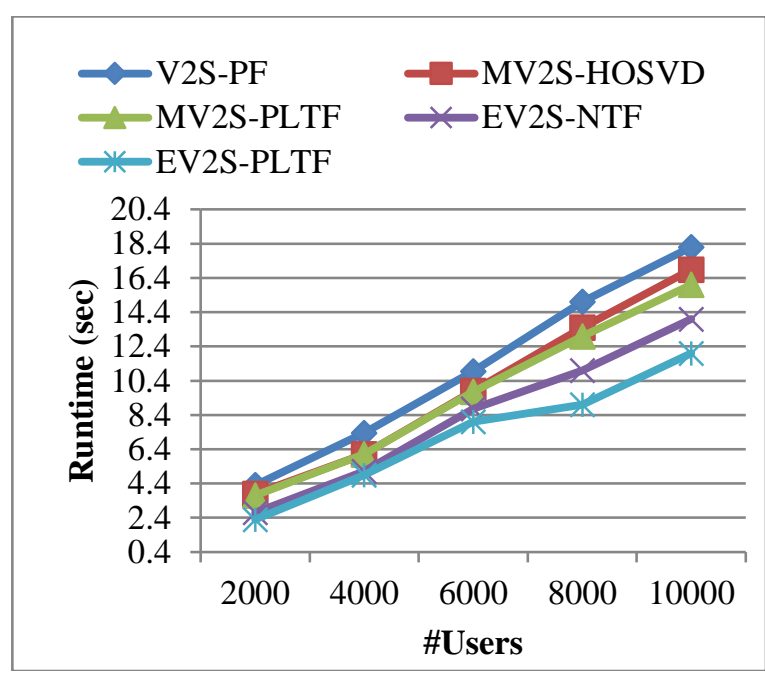

Figure 3a: Runtime Vs Users

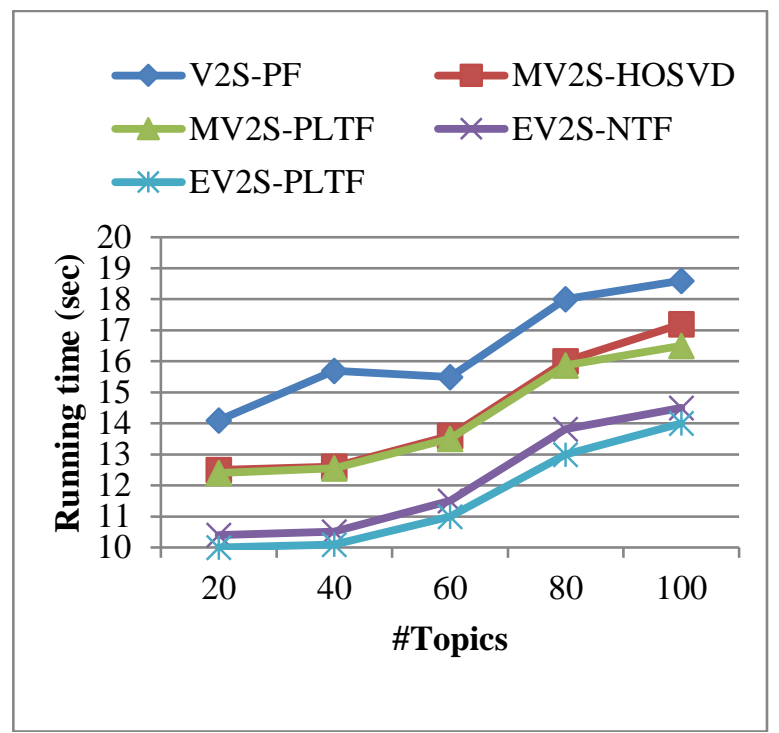

Figure 3b: Runtime Vs Topics

Figure 3 a) \& b) depicts the runtime evaluation of the factorization techniques in contradiction of the amount of users and amount of topics correspondingly. It is proved that the presented EV2S-NTF, EV2S-PLTF based factorization approaches contain minimum runtime compared to the $\mathrm{MV} 2 \mathrm{~S}$ and $\mathrm{V} 2 \mathrm{~S}$ based factorization approaches. Therefore from the outcomes it is proved that the EV2S-NTF, EV2S-PLTF based factorization approaches does superior in micro-blogging content propagation modeling with greater values of performance metrics.

\section{Conclusion and future work}

This work presented Enhanced V2S factorization framework by uniting Probabilistic Latent Tensor Factorization (PLTF) techniques and Non-negative Tensor Factorization (NTF). This model takes linguistic features in the network as a behavior factor in the company of the other factors called user's topic virality, position, user susceptibility and user virality. By means of presented EV2S-NTF and EV2S-PLTF technique, Data 
representation for tensor factorization is improved very proficiently. It initially produces improved tensors and after that factorizes the tensors concurrently over the EV2S model.

The most followed content is guaranteed by means of user's position as well as the finest linguistic features. The improved tensor factorization framework enhances the identification of content propagation. Stochastic Gradient Descent (SGD) technique is presented for resolving the unrestrained optimization issue. It is a stochastic guesstimate of the gradient descent optimization technique for reducing an objective function. The experimentation outcomes prove that by means of the research model, improved content propagation modeling is attained. As a result the research model is extremely effective, while explored extra choices it is identified that the linguistic patterns in the tweets participate significantly to the content propagation modeling. Henceforth, sentiments are regarded to implement the overall system performance. As well, it could expand the SGD method that needs previous acquaintance regarding the uneven data to estimate the optimization issue.

\section{References}

[1] Y1lmaz YK \& Taylan Cemgil A, “Algorithms for probabilistic latent tensor factorization”, Signal Processing, Vol.92, No.8 (2012), pp. $1853-1863$

[2] Leskovec J, Adamic LA \& Huberman BA, "The dynamics of viral marketing", ACM Transactions on the Web (TWEB), Vol.1, No.1, (2007).

[3] Scott J, "Social network analysis: developments, advances, and prospects", J Soc Netw Anal Min (SNAM), (2011).

[4] Sun E, Rosenn I, Marlow C \& Lento T, "Gesundheit! Modeling contagion through Facebook news feed", Proceedings of AAAI international conference on weblogs and social media, (2009).

[5] Lakshmi, K., Surendar, A. "Verification of axiprotocol using system Verilog", (2017), International Journal of Mechanical Engineering and Technology, 8 (5), pp. 588-595.

[6] Gupte M, Hajiaghayi M, Han L, Iftod L, Shankar P \& Ursu RM, "News posting by strategic users in a social network", Proceedings of international workshop on internet and network economics, (2009).

[7] Kempe D, Kleinberg J M \& Tardos E, "Maximizing the spread of influence through a social network", Proceedings of ACM SIGKDD International Conference on Knowledge Discovery and Data Mining, (2003).

[8] Ma H, Yang H, Lyu MR \& King I, "Mining social networks using heat diffusion processes for marketing candidates selection", Proceedings of ACM international conference on information and knowledge management, (2008).

[9] Mu Y, Ding W, Zhou T \& Tao D, "Constrained stochastic gradient descent for large-scale least squares problem", Proceedings of the 19th ACM SIGKDD international conference on knowledge discovery and data mining, (2013), pp.883-891.

[10] Baeza-Yates R \& Ribeiro-Neto B, "Modern Information Retrieval". Addison-Wesley, Reading, MA, USA, (1999).

[11] Baumgartner R, Flesca S \& Gottlob G, "Visual Web information extraction with Lixto", Proceedings of the $27^{\text {th }}$ International Conference on Very Large Databases, (2001), pp119-128.

[12] Lerman K \& Ghosh R, "Information contagion: An empirical study of the spread of news on Digg and Twitter social networks", ICWSM, Vo.10, (2010), pp.90-97.

[13] Wu S, Hofman JM, Mason WA \& Watts DJ, "Who says what to whom on twitter", Proceedings of the 20th international conference on World wide web, (2011), pp.705-714

[14] Suh B, Hong L, Pirolli P \& Chi EH, "Want to be retweeted? large scale analytics on factors impacting retweet in twitter network", IEEE second international conference on Social computing (socialcom), (2010), pp.177-184.

[15] Petrovic S, Osborne M \& Lavrenko V, "RT to Win! Predicting Message Propagation in Twitter", ICWSM, (2011).

[16] Vishnu, S., Vignesh, S., Surendar, A.’Design and implementation of ZETA micro-inverter for solar PV application"(2017), International Journal of Mechanical and Production Engineering Research and Development, 7 (4), pp. 215-222.

[17] Romero DM, Meeder B \& Kleinberg J, "Differences in the mechanics of information diffusion across topics: idioms, political hashtags, and complex contagion on twitter", Proceedings of the 20th international conference on World wide web, (2011), pp. $695-$ 704.

[18] Mukherjee S \& Pushpak B, "Sentiment Analysis in Twitter with Lightweight Discourse Analysis", COLING, (2012).

[19] Shashua A \& Hazan T, "Non-negative tensor factorization with applications to statistics and computer vision", Proceedings of the 22nd international conference on Machine learning, (2005), pp. 792-799.

[20] Hazan T, Polak S \& Shashua A, "Sparse image coding using a 3D non-negative tensor factorization", Tenth IEEE International Conference on Computer Vision, Vol.1, (2005), pp.50-57.

[21] Cemgil AT, Şimşsekli U \& Sübakan YC, "Probabilistic latent tensor factorization framework for audio modeling", IEEE Workshop on Applications of Signal Processing to Audio and Acoustics, (2011), pp.137-140.

[22] Hoang TA \& Lim EP, "Microblogging Content Propagation Modeling Using Topic-specific Behavioral Factors", IEEE Transactions On Knowledge And Data Engineering, (2016). 